\title{
Stimulation of the Olfactory Epithelium with Odorants in Gaseous and Aqueous Phases
}

\author{
Masae IINo and Sadayuki F. TAKAGI \\ Department of Physiology, School of Medicine, \\ Gunma University, Maebashi, 371 Japan
}

\begin{abstract}
The olfactory epithelia of the bullfrog and the carp were stimulated with various odorants in the gaseous and/or aqueous phases. Some ionic solutions were also applied.

1. By recording the induced waves in the olfactory bulb, it was shown that the bullfrog responds to odorants in the two phases, but the carp can respond only to odorants in the aqueous phase.

2. Comparison of the stimulative effects of the same odorants in the two phases were made in the bullfrog. Although various response patterns to gaseous and aqueous stimuli of single cells in the olfactory bulb were found in most cases, a difference in the application methods of vapors and solutions made such comparison difficult.

3. The same single cells in the olfactory bulb usually responded to various odorous vapors and solutions as well as to ions in different patterns. When the responses to the vapors and solutions of the same odorants of different concentrations were compared, different patterns were also found.
\end{abstract}

In two previous papers (TAKAGI et al., 1978a, b), it was shown that the olfactory epithelia of the bullfrog and the carp respond not only to odorous substances, but also to bitter, salty and acid taste stimuli and they also respond well to various cations and anions. In these experiments, the induced waves recorded from the olfactory bulb were mainly used as indicators of the olfactory responses.

In the present experiment, the problems of whether or not a bullfrog, a terrestrial animal can respond to odors in the aqueous phase, and conversely whether or not a carp does to odors in gaseous phase were investigated. Applying odorants of different concentrations in gaseous and aqueous phases and also some odorous and ionic solutions, responses of single cells in the olfactory bulb were recorded. Thus, discriminative mechanism of odors and ions was studied in the olfactory bulb.

Received for publication July 19, 1977

飯野昌枝, 高木貞敬 


\section{MATERIALS AND METHODS}

Animals. The bullfrog, Rana catesbeiana, and the carp, Cyprinus caprio, were used. Animals were immobilized by the same methods as in two previous papers (TAKAGI et al., 1978a, b).

Stimuli. Butyl alcohol, methyl acetate and ethyl ether were used. The original solutions of the three odorous substances were diluted with distilled water and $1 \%, 0.5 \%$ and $0.25 \%$ solutions were prepared. One cc of each solution was stored in a $30 \mathrm{ml}$ syringe and the vapor inside the syringe was blown onto the olfactory epithelium as a gaseous stimulant. As an aqueous stimulant, one or two drops of each solution was dripped onto the olfactory epithelium. Isotonic $\mathrm{Rb}^{+}(115 \mathrm{mM})$ and $\mathrm{Sr}^{2+}(84.4 \mathrm{mM})$ solutions, quinine hydrochloride $(5 \times$ $10^{-2} \mathrm{M}$ ) solution and distilled water were used as aqueous stimulants. After a gaseous stimulant was applied, a purified air was always blown in puffs onto the olfactory epithelium in order to remove the remaining stimulant. After an aqueous stimulant was dripped, the olfactory epithelium was always rinsed with an isotonic $(6 \%)$ sucrose solution.

Recording. Induced waves and single cell discharges of the olfactory bulb were recorded also by the same procedures as before (TAKAGI et al., 1978a).

\section{RESULTS}

(1) Stimulations with odorants in gaseous and aqueous phases in a terrestrial animal and a fish

In some of our previous experiments, saturated and diluted vapors of butyl alcohol, methyl acetate and ethyl ether were applied to the olfactory epithelium of the bullfrog and remarkable induced waves were recorded in the olfactory bulb ( 1 and 3 in Fig. 1). However, solutions of these substances were never applied in the earlier experiments. When $1 \%$ solutions of them were applied to the olfactory epithelium of the bullfrog, induced waves appeared strikingly in the olfactory bulb ( 2 and 4 in Fig. 1). Boiled solution of the silkworm pupae, which was often used as an olfactory stimulus in the fish (SHIBUYA, 1960), was also found to work as an effective olfactory stimulus to the bullfrog (5 in Fig. 1). Thus, it is clear that the olfactory epithelium of the bullfrog can be stimulated not only by odorous vapors but also by odorous solutions.

In the olfactory epithelium of the carp, the same experiments were repeated. These odorous solutions were found to work as effective stimuli (6 and 7 in Fig. 1). When these odorous vapors were applied, however, induced waves did not appear in the olfactory bulb of the carp. Consequently, it was shown in the olfactory epithelium of the carp that odorous solutions can be effective stimuli, but odorous vapors are not. Thus, a difference was found between the olfaction of a terrestrial animal and a fish. 


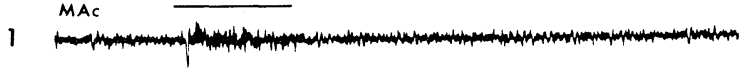

2

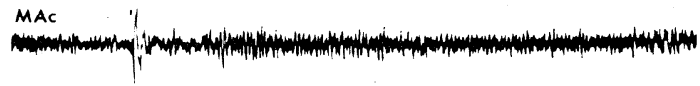

3
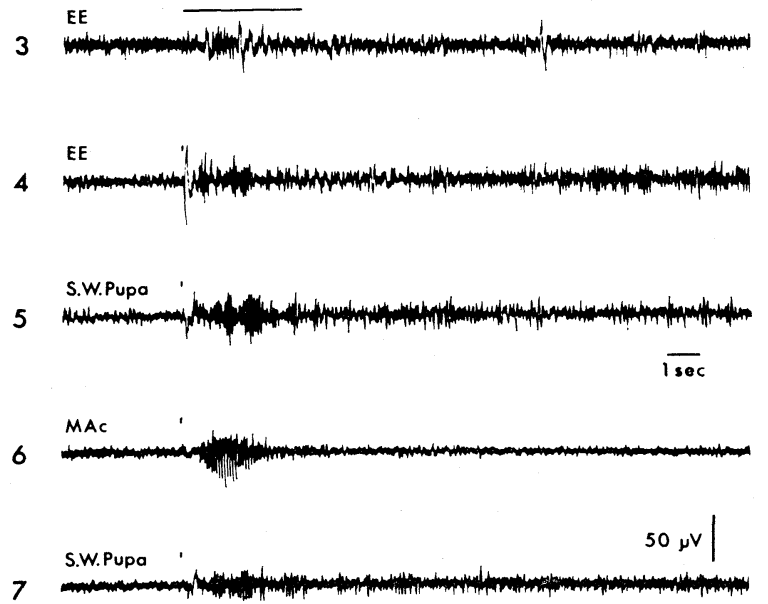

$\overline{1 \mathrm{sec}}$

Fig. 1. Stimulation with odors in gaseous and aqueous phases. $1 \%$ vapors of methyl acetate (MAc) and ethyl ether (EE) were applied to the olfactory epithelium of the bullfrog in 1 and $3.1 \%$ aqueous solutions of methyl acetate and ethyl ether were dripped to the olfactory epithelium of the bullfrog in 2, 4 and to that of the carp in 6 . Boiled water of the silkworm pupae was similarly dripped to the epithelium in the bullfrog in 5 and in the carp in 7. In each case, induced waves were observed. But when odorous vapors were applied to the olfactory epithelium of the carp, no induced wave appeared.

(2) Comparison of the effects of the same odorants in gaseous and aqueous phases As shown above, the induced waves were elicited in the olfactory bulb of the bullfrog by the application of several odors in gaseous and aqueous phases. However, a question as to whether or not the same olfactory sensation is elicited by the same odorants in both phases has been entirely unanswered. In order to answer the question, recording of single olfactory cell responses was tried in the olfactory epithelium, while many stimuli were successively applied in both phases. However, it was found to be extremely difficult, and, therefore, responses of single cells in the olfactory bulb were examined. Vapors and solutions of $1 \%$ butyl alcohol, $1 \%$ methyl acetate, $1 \%$ ethyl ether and $12.5 \mathrm{~mm}$ ethyl $n$-butyrate were applied in succession to the olfactory epithelium. Spontaneous discharges of single cells decreased or disappeared for various durations in response to some 
odorants (inhibition type), but increased for short or long durations to some other odorants (facilitation type). To still other odorants, spontaneous discharges did not show any change (no-response type). When the results were compared, spontaneous discharges were facilitated or inhibited in parallel by odorants in both phases, but in other cases, they were facilitated by odorants in one phase, and inhibited by the same odorants in the other phase or vice versa (Fig. 2).

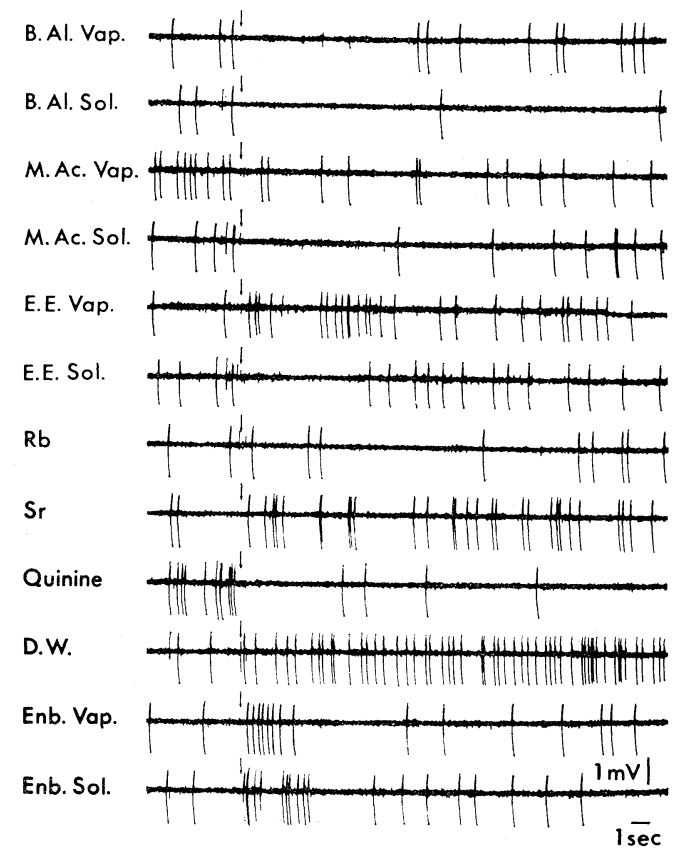

Fig. 2. Responses of single cells to various stimuli, recorded from the olfactory bulb. To the olfactory epithelium of the bullfrog $1 \%$ vapors (Vap) and $1 \%$ aqueous solutions (Sol) of butyl alcohol (B. Al.), methyl acetate (M. Ac.), ethyl ether (E. E.) and $12.5 \mathrm{~mm}$ ethyl $n$-butyrate $(\mathrm{Enb})$ were applied. Moreover, isotonic solutions of $\mathrm{Rb}^{+}(115 \mathrm{~mm})$ and $\mathrm{Sr}^{2+}(84.4 \mathrm{mM}), 5 \times 10^{-2} \mathrm{M}$ quinine hydrochloride solution and distilled water (D.W.) were also dripped.

The response types were different from cell to cell. Response types of "facilitation followed by inhibition" and of "inhibition followed by facilitation" were also found. Besides, the same cells showed complicated combinations of many response types to $\mathrm{Rb}^{+}, \mathrm{Sr}^{++}$, bitter-tasting solution, and distilled water (Fig. 2). All the responses of 40 bulbar cells were arranged according to the increasing number of effective stimuli in Fig. 3. Among the 40 cells there were not found two single cells which responded with the same pattern to the same kinds of stimuli. 


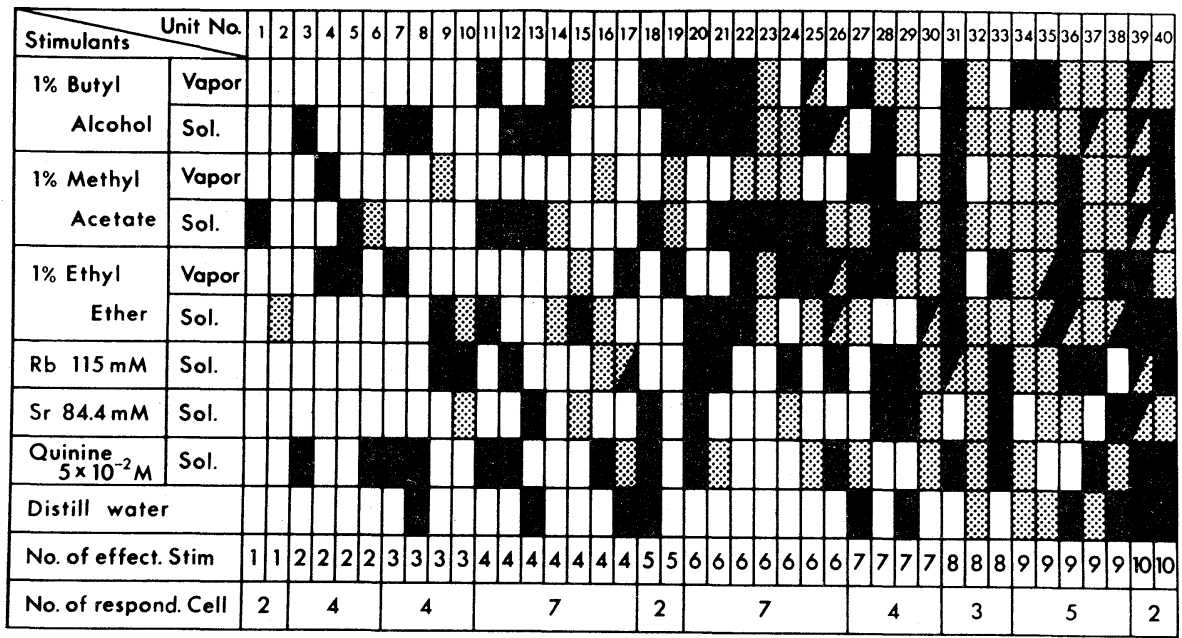

Facilitatory

Inhibitory

Facilitation followed by Inhibition

Inhibition followed by Facilitation No response

Fig. 3. Response types of 40 single cells in the olfactory bulb to various odors in gaseous and aqueous phases. Some cells responded to one to six stimulants with facilitation type only. Some other cells responded with facilitation type to some odorants but with inhibition type to other odorants. Moreover, response types of "facilitation followed by inhibition" or "inhibition followed by facilitation" were also observed in some cases together with simple facilitation and/or inhibition types. The same stimulants as in Fig. 2 were used except for ethyl $n$-butyrate. 'No. of effect. Stim' means number of the effective stimuli which induced some change in the spike discharges of single cells. 'No. of respond. Cell' means number of cells which showed responses of the indicated numbers to the stimuli applied.

(3) Stimulation with odorants of different concentrations in gaseous and aqueous phases

The same odors, butyl alcohol, methyl acetate and ethyl ether were applied at the three concentrations of $0.25 \%, 0.5 \%$ and $1 \%$ in gaseous and aqueous phases.

When the odors were applied with increasing concentrations, single cells in the olfactory bulb often showed the same response types in spite of the difference in concentrations. But in many cases, the response types changed with increase in concentrations, and sometimes parallel changes were produced by the gaseous and aqueous stimuli (Fig. 4). Besides, with increase in concentration were found shifts of the response pattern from no-response-type through facilitation-type to inhibition-type (in case of aqueous stimulation in Fig. 5), from inhibition-type to facilitation-type (in case of gaseous stimulation in Fig. 4), from no-responsetype to facilitation- or inhibition-types or vice versa, from facilitation-types to inhibition- and/or no-response-types or vice versa, and etc. Among them, a type of inhibition to an odor of a lower concentration and facilitation to that of a higher 


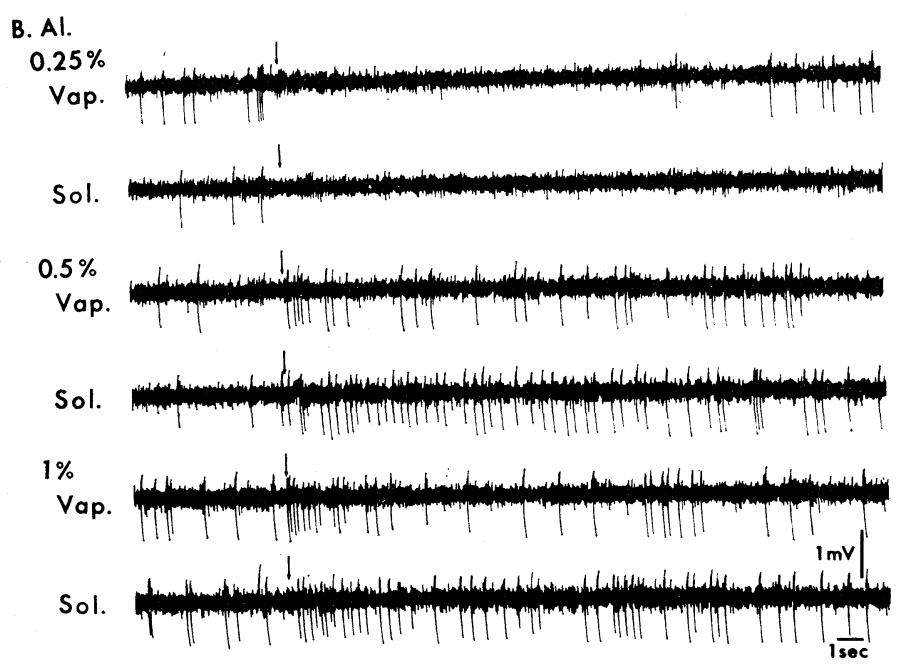

Fig. 4. Stimulation with butyl alcohol of three concentrations in gaseous and aqueous phases. In this case, spontaneous discharges were inhibited by $0.25 \%$ vapor and solution, increased by the vapors and solutions of $0.5 \%$ and $1 \%$. Thus, the responses to the odorants in the two phases appeared in parallel with the same patterns.

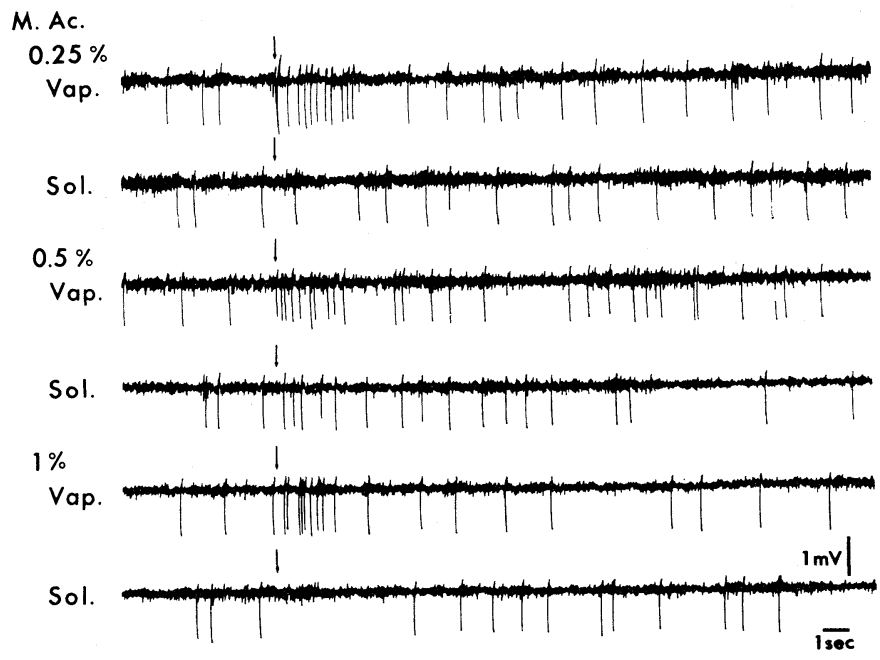

Fig. 5. Stimulation with methyl acetate of three concentrations in gaseous and aqueous phases. In this case, spontaneous discharges increased to $0.25 \%$ vapor (facilitation type) but did not show any change to $0.25 \%$ solution (no response type). Similar increase was found to $0.5 \%$ and $1 \%$ vapors (facilitation type). On the other hand, $0.5 \%$ solution slightly increased spontaneous discharges (facilitation type), but $1 \%$ solution inhibited it for a short time (inhibition type). 
concentration was noteworthy, because responses of the reversed type, namely, facilitation to a weak odor and inhibition to a stronger odor is supposed to be rather common. This seemingly paradoxical response type may be attributable to some complex neuron circuits in the olfactory bulb. Response types of "facilitation followed by inhibition" or "inhibition followed by facilitation" were also found. All the response types to the three odors in three different concentrations are summarised in Fig. 6.

1

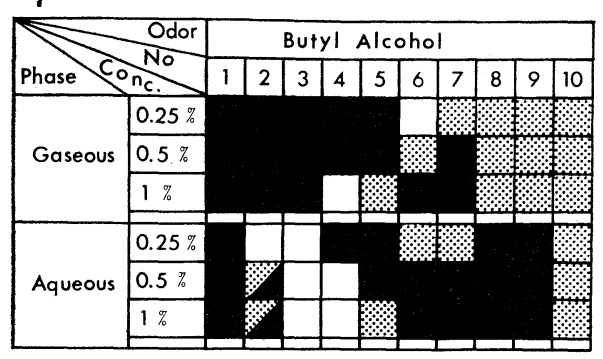

2

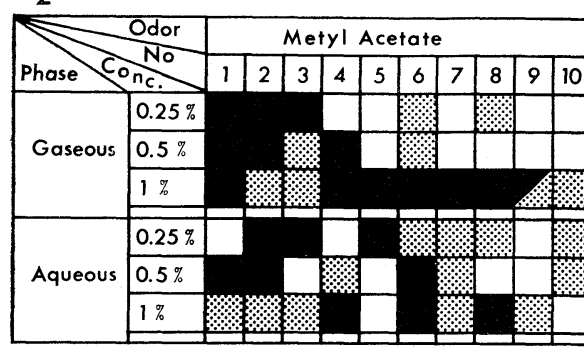

3

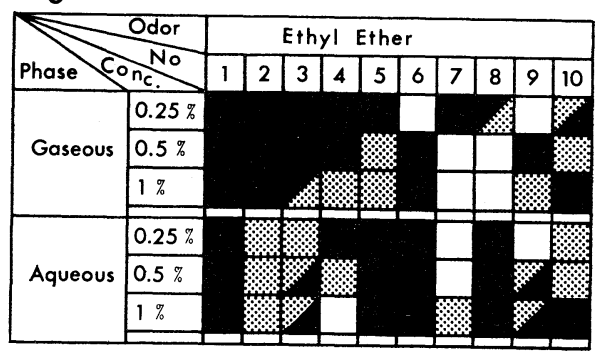

Facilitatory Inhibitory $\square$ No response

Facilitation followed

Inhibition followed

by Facilitation

Fig. 6. Response types of 10 single cells in the olfactory bulb to three odorants of three concentrations in gaseous and aqueous solutions. To odorants of three concentrations, a few cells responded with the same patterns, but many other cells responded with different patterns. Two cells showed no-response-, facilitation- and inhibition-types to stimuli of increasing concentrations, but other cells showed complicated response patterns.

When the responses to odors in the gaseous phase are compared with those to the same odors of the same concentrations in the aqueous phase, entirely the same response patterns were found in some cases, but very different response patterns were observed in other cases. Since odorous vapors were blown in puffs while odorous solutions were dripped, these difference in the response patterns may be partly or mainly due to the difference in the method of application, even if the same odorous substances were applied in the same concentrations.

\section{DISCUSSION}

(1) Difference in olfaction between a terrestrial animal and a fish. The present experiments demonstrated a fact that the olfactory epithelium of the bullfrog 
responds to odorants in the aqueous phase as well as in the gaseous phase, while the olfactory epithelium of the carp did only to odorants in the aqueous phase.

The carp is phylogenetically in a lower position in comparison to the bullfrog, and lives in water during its entire life, exposing the olfactory epithelium to water but never to air. The adult bullfrog lives as a terrestrial animal, exposing its olfactory epithelium to air all the time, but its tadpole lives only in water just like a fish, exposing the olfactory epithelium to water. The finding that the adult bullfrog can also smell odors in water is explicable if it is assumed that the animal retains the olfactory property acquired during the larval stage for its entire life.

However, the olfactory epithelium of the dog also responds to a $\mathrm{NaCl}$ solution (UEKI and Domino, 1961). Consequently, it is highly probable that the property to respond to odorants in water is retained not only in the amphibia, but also in phylogenetically higher animal species. Thus, a difference was found between the olfactory receptive mechanisms of a terrestrial animal and a fish. It is still entirely open to question as to what kind of structural difference in the olfactory receptive sites of the two kinds of animals causes this difference in response.

(2) Comparative study of gaseous and aqueous stimulation with the same odorants. The response patterns of single cells to various odorants either in the gaseous phase or in the aqueous one were studied in the olfactory bulb by many investigators (ShiBUYA et al., 1962; DövING, 1965, 1966a, b; Döving et al. 1974; HARA, 1967, 1975; HigaShino et al., 1969; MATHEWs 1972a, b; TANABE et al., 1975; SHIBUYA et al., 1977). In the present experiment, odorous substances of the same concentrations were applied in the gaseous and aqueous phases, and the stimulative effects were compared. Such a comparative study as was performed here has never been made before. The response patterns in the two phases were found different in most cases. However, a difference exists in the method of application between gaseous and aqueous odorants. Such a difference of methods very probably makes it difficult to strictly compare the stimulative effects of the same odorants in the two phases. Therefore, it is probably impossible to determine whether or not the same odorous substances of the same concentrations in the two phases can elicit the same olfactory sensation.

(3) Discrimination of olfactory stimuli in the olfactory bulb. The response patterns of single cells in the olfactory bulb were studied applying various kinds of stimuli in gaseous and aqueous phases (Fig. 3). The results reveal that each single cell in the olfactory bulb responds differently to stimuli in most cases and that no two cells respond in the same pattern.

Next, when the concentrations of odorous substances in the gaseous and aqueous phases are increased from $0.25 \%$ to $1 \%$, the response patterns in many cases were different depending upon the concentrations of stimuli (Fig. 6).

Considering all these results, it is clear that cells in the olfactory bulb on the whole respond differently to different odorants, to the same odorants of different concentrations in the gaseous and aqueous phases, and also to ions. These 
differences in the responses may form an important basis of olfactory discrimination.

We are indebted to Miss T. Yajima and Mrs. T. Wada for preparing the figures.

This work was supported by a grant for scientific research from the Ministry of Education, Science and Culture of Japan.

\section{REFERENCES}

Döving, K. (1965) Studies on the responses of bulbar neurons of frog to different odour stimuli. Rev. Laryngol. (Bordeaux), 86: 845-854.

Döving, K. (1966a) An electrophysiological study of odour similarities of homologous substances. J. Physiol. (Lond.), 186: 97-109.

Döving, K. (1966b) Analysis of odour similarities from electrophysiological data. Acta Physiol. Scand., 68: 404-418.

Döving, K. B., Nordeng, H., and OAKLeY, B. (1974) Single unit discrimination of fish odours released by char (Salmo alpinus L.) Populations. Comp. Biochem. Physiol., 47A: 1051-1063.

HARA, T. J. (1967) Electrophysiological studies of the goldfish, Carassius auratus L. II. Response patterns of the olfactory bulb neurons to chemical stimulation and their centrifugal control. Comp. Biochem. Physiol., 22: 199-208.

Hara, T. J. (1975) Molecular structure and stimulatory effectiveness of amino acids in fish olfaction. In: Olfaction and Taste V, ed. by Denton, D. A. and Coghlan, J. P. Academic Press, New York, pp. 223-225.

Higashino, S., TAKeuchi, H., and Amoore, J. E. (1969) Mechanism of olfactory discrimination in the olfactory bulb of the bullfrog. In: Olfaction and Taste III, ed. by PFAFFMAN, C. Rockefeller Univ. Press, New York, pp. 192-211.

Mathews, D. F. (1972a) Response patterns of single units in the olfactory bulb of the rat to odor. Brain Res., 47: 389-400.

MATHEWS, D. F. (1972b) Response patterns of single neurons in the tortoise olfactory epithelium and olfactory bulb. J. Gen. Physiol., 60: 166-180.

SHibuYA, T. (1960) The electrical responses of the olfactory epithelium of some fishes. Jap. J. Physiol., 10: 317-326.

ShibuYa, T., AI, N., and TaKagi, S. F. (1962) Response type of single cells in the olfactory bulb. Proc. Japan Acad., 38: 231-233.

Shibuya, T., Aihara, Y., and Tonosaki, K. (1977) Single cell responses to odors in reptilian olfactory bulb. In: International Symposium on Food Intake and Chemical Senses, ed. by Katsuki, Y. Univ. Tokyo Press, Tokyo, pp. 23-32.

TAKAGI, S. F., IINo, M., and YarITA, H. (1978a) Effects of gustatory stimulants upon the olfactory epithelium of the bullfrog and the carp. Jap. J. Physiol., 28: 109-128.

TAKagi, S. F., IINO, M., YARITA, H., and Mori, K. (1978b) Ionic stimulation of the olfactory epithelium in the bullfrog and the carp. Jap. J. Physiol., 28: 129-148.

TAnabe, T., IINo, M., and TAKagi, S. F. (1975) Discrimination of odours in olfactory bulb, pyriform-amygdaloid areas, and orbitofrontal cortex of the monkey. J. Neurophysiol., 38:
1284-1296.

UeKI, S. and Domino, E. F. (1961) Some evidence for a mechanical receptor in olfactory function. J. Neurophysiol., 24: 12-25. 